\title{
Psychosocial interventions for adults with visible differences: A systematic review
}

Alyson Norman, Timothy P Moss

Background: Some individuals with visible differences have been found to experience psychosocial adjustment problems that can lead to social anxiety and isolation. Various models of psychosocial intervention have been used to reduce social anxiety and appearance related distress in this population. The objective of this review was to update a previous systematic review assessing the efficacy of psychosocial intervention programs for adults with visible differences. The original review (Bessell \& Moss, 2007) identified 12 papers for inclusion. Methods: A search protocol identified studies from 13 electronic journal databases. Methods: Studies were selected in accordance with pre-set inclusion criteria and relevant data were extracted. Results: This update identified an additional four papers that met the inclusion criteria. Two papers provided very limited evidence for the efficacy of a combined cognitive-behavioural and social skills training approach. None of the papers provided sufficient evidence for the optimal duration, intensity or setting of psychosocial interventions for this population. Discussion: The review concluded that a greater number of Randomised Controlled Trials and experimental studies were required to increase the methodological validity of intervention studies. 


\section{Author Details:}

3 Norman, Alyson ${ }^{1}$ (corresponding author): Alyson.norman@plymouth.ac.uk, tel: +44(0)1752 584844.

4 Moss, Timothy.P2.tim.moss@uwe.ac.uk

5

6 1: School of Psychology, Plymouth University, Portland Square, Drake Circus, Plymouth, UK, PL4

7 8AA

8 2: Centre for Appearance Research, University of the West of England, Frenchay Campus,

9 Coldharbour Lane, Bristol, UK, BS16 1QY.

\section{Acknowledgements}

11 Thanks to Julie Griffin, Natasha-Ann Deeprose, Hannah Wadland, Amir Massoumian and Jo-Lee

12 Hankinson for support with title and abstract checking and risk of bias assessment. 


\section{Introduction}

15 The term visible difference refers to any kind of condition, whether congenital or acquired that can

16 leave an individual with an altered appearance (e.g. skin conditions, burns, scarring or craniofacial

17 abnormalities. Some individuals with visible differences have been found to experience psychosocial

18 adjustment problems that can lead to social anxiety and isolation (Rumsey et al, 2004; Rumsey \&

19 Harcourt, 2012) and poor quality of life (Marcusson, Paulin \& Ostrup, 2002). As such, appearance

20 altering conditions present a clear challenge to a positive body image for those affected and have led

21 to the development of numerous psychosocial intervention programs designed to address the

22 psychological, as well as the physical needs and difficulties experienced by those with visible

23 differences. The psychosocial difficulties experienced by some of those with visible differences

24 include name calling, staring and unsolicited questioning about their appearance (Kleve \& Robinson, 25 1999).

There are many different models that outline the difficulties experienced by some individuals

27 with visible differences. These include the social anxiety model (Baumeister \& Leary, 1995),

28 Goffman's (1968) model of stigma, social skills models (Bull \& Rumsey, 1988) and models of body

29 image disturbance (Cash, 2001). Baumeister and Leary's (1995) model suggests that individuals with

30 visible differences experience social anxiety at least in part because they are fearful of being rejected

31 or excluded on the grounds of having an unusual or different appearance (Kent, 2000). Therefore, this

32 model suggests that it is important to focus interventions on reducing social anxiety through exposure

33 to social situations in order to promote positive adjustment amongst those with visible differences

34 (Newell \& Marks, 2000). Goffman's (1968) stigma model fits in many ways with the social anxiety

35 model, and states that having a different appearance is a characteristic that is "devalued" by society 
36 and as such those with a visible difference are more likely to be excluded or rejected, which suggests

37 a very real reason for experiencing social anxiety. Some research has suggested that those with visible differences can become preoccupied with

39

40 conform to the cultural norms of attractiveness that their society imposes. This social pressure to look

52 a certain way, alongside a more personal form of stigma, where they themselves feel they should look

53 "normal", can lead to high levels of body image disturbance, which is associated with poorer

54 adjustment (Altabe \& Thompson, 1996). This model suggests that interventions should focus

55 specifically on addressing the way individuals feel about their appearance and the negative

56 assumptions they make about the importance of appearance. 
The reality is that no one model completely explains the experience of living with a visible

58 difference. Kent (2000) recommended an integrated model that addresses body image dissatisfaction

59 and the negative assumptions associated with appearance concerns. He also suggested that it is

60 important to target social anxiety with exposure therapy (introducing people to feared social

61 situations). However, as there is a very real tendency for individuals to experience negative responses

62 from others, it is important to boost social skills too, in order to provide individuals with the

63 techniques that they will need to deal with these responses. Both social skills training (SST) and

64 cognitive behavioural therapy (CBT) are common intervention types for adults with visible

65 differences.

66 Although these intervention techniques for people with a visible difference are used, there is

67 still a significant lack of evidence pertaining to the efficacy of these different psychosocial techniques.

68 A systematic review conducted by Bessell and Moss (2007) found little to no evidence to support any

69 particular intervention model, due to methodological constraints associated with the included

70 studies.

A recent systematic review conducted by Muftin and Thomson (2013) looked at self-help

72 psychosocial interventions for individuals with visible differences. Whilst this is an important update,

73 the review does not incorporate all forms of psychosocial intervention, only those administered in a

74 self-help format. Additionally, the interventions did not necessarily measure body image

75 dissatisfaction or appearance-related distress which is a key variable that can identify level of distress

76 in the study of visible difference (Clarke et al, 2008). Therefore the review does not help to answer

77 fundamental questions raised by the original review regarding method of delivery (Bessell \& Moss,

78 2007). Furthermore, it is imperative that psychosocial interventions are employed using an evidence- 
79 based practice approach (Anderson, 2006). For that reason it is important that the original review be

80 updated to ensure an accurate evidence base for psychosocial interventions for this population. It is

81 therefore, the belief of the current authors that this update is both needed and timely.

\section{Objectives}

83 The aim of the present study is to assess the evidence-base for psychosocial intervention programs

84 for adults with visible differences with a view to reducing appearance-related distress, body image

85 dissatisfaction. This will be done through an update of the existing systematic review (Bessell \& Moss,

86 2007) from 2006 (the date of the last search) to the present day. Where appropriate, meta-analysis

87 was used to synthesise findings across papers. The overall intention of this study was to identify the

88 evidence base for the therapeutic technique employed, as well as the method of delivery and optimal

89 length and intensity of therapy.

\section{Methods}

\section{Study selection}

92 The search aimed to identify all studies relating to psychosocial interventions for adults with visible

93 differences from January 2006 (six months prior to the original search in Bessell \& Moss, 2007) to $12^{\text {th }}$

94 May 2014. An extensive search strategy was used to search 13 databases, including Medline, embase,

95 psychinfo, and Cochrane central register of Controlled trials (CENTRAL) (See Appendix A for full search

96 strategy). This was compiled by a library technician based on an exhaustive list of appearance altering

97 conditions and types of psychosocial intervention. No language restrictions were applied. In addition

98 websites including National Institute of Clinical Excellence (NICE) and the metaRegister of Controlled 
99 Trials (mRCT) were searched and reference lists of included papers. Search criteria were adapted to

100 suit the search terms of each individual database.

102 Inclusion criteria

103 Study design: No exclusions were applied based on study design with all study designs being included

104 in the review. Case studies with less than five participants in each group were excluded.

105 Population: Adults with noticeable visible differences, e.g. disfigurements of face, neck and hands.

106 This included a wide range of different conditions from congenital skin conditions and abnormalities

107 to cancer patients, or those with scars resulting from injury. All client groups were over the age of 16 .

108 Both males and females of any ethnicity or race were included.

109 Interventions: These included CBT, SST and more traditional forms of psychotherapy all delivered

110 either alone or as part of a package of care. The interventions had to include some element

111 specifically designed to target appearance concerns.

112 Comparators: The comparators used in this review were current standard treatments including

113 standard therapist-led CBT for the treatment of anxiety or depression, non-directive counselling,

114 primary care counselling, routine management (drug treatments for anxiety or depression) and no

115 treatment.

116 Outcomes: The primary outcome measure was any measure of appearance related distress (e.g. body

117 image concerns, body image quality of life etc.). Only studies with this primary outcome measure

118 were included in the review ${ }^{1}$. Secondary measures included measure of anxiety and depression and

\footnotetext{
1 The aim of this review was specifically to assess appearance-related distress not general psychosocial
} functioning and differs to other reviews, e.g. Muftin \& Thompson, 2013. 
119 general improvements in psychological symptoms, interpersonal and social functioning, satisfaction

120 and preference, site of delivery and acceptability of treatment.

\section{Exclusion Criteria}

122 Any treatment designed to treat body dysmorphic disorder or eating disorders such as bulimia

123 nervosa or anorexia nervosa were excluded. It was also decided to exclude any visible differences that

124 were not considered to be commonly on display (such as breast reconstruction, abdominal injury).

125 These types of conditions do fall within the remit of visible differences, but it was considered that the

126 intervention needs of individuals with "hidden" differences might be different to those with normally

127 visible differences, meaning that different intervention techniques may be appropriate. For example

128 social skills models are often used to help individuals manage questioning around appearance (Kent

129 2000). This particular model may be less helpful to those with hidden differences who do not

130 experience unsolicited questioning.

\section{Analysis}

133 The authors used a qualitative approach to synthesise data across studies (Dixon-Woods et al., 2005)

134 and focused on three main areas: information pertaining to theoretical or therapeutic perspective,

135 method of delivery (setting, person delivering the intervention) and timing of the intervention

136 (intensity and frequency of the intervention).

138 Meta-analysis of Trials only 
139 Outcome Measures: Primary and secondary outcome measures of psychosocial adjustment were

140 extracted (e.g. preoccupation with appearance, anxiety, depression, confidence, quality of life, social

141 integration).

142 Effect Sizes: Standard mean differences (SMDs) and/or effect sizes together with 95\% confidence

143 intervals (Cls) were extracted for continuous outcomes and odds ratios (ORs) together with 95\% Cls

144 were extracted for dichotomous outcomes. Meta analyses were only conducted on multiple

145 randomised controlled trials (RCTs) of similar interventions to allow appropriate data pooling.

\section{Assessment of risk of bias}

147 Three reviewers (AN, AM \& JG) independently assessed trials using the Cochrane Risk of Bias tool

148 (Higgins \& Green, 2011). In the case of observational studies two reviewers (AN \& JG) used the

149 RAMbo assessment tool (Chen \& Wang, 2009) to assess the quality of randomization (R), whether

150 missing data was accounted for (A) and whether the type of measurement was appropriate (M).

152 Results

153 The search results identified 13837 possible studies since the previous review was conducted. After

154 removal of duplicates, 3539 studies were identified for further investigation. Of these 3468 studies

155 were identified as not relevant for inclusion within the review and were discarded on the basis of

156 titles and abstracts independently by at least two reviewers (AN, AM \& JG). This left 71 studies to

157 assess for inclusion (See figure 1).

158 Insert figure 1 here

159 Of the 71 papers identified for possible inclusion, four met the inclusion criteria on closer inspection 160 by three reviewers (AN, TM \& AM). Sixty-seven studies were excluded. Reasons for exclusion included 
161 studies that did not assess an intervention targeting appearance or related psychosocial distress (27

162 studies), those that did not assess an intervention (five studies), case studies with less than 5

163 participants in each group (13 studies), descriptive articles or review papers (14 papers), those with

164 no primary outcome measure of appearance-related distress or body image concern (6 studies) and

165 two which met the inclusion criteria, but not enough data was present in the abstracts to include

166 within the review (authors were contacted for full papers but were not supplied).

\section{Risk of Bias Assessment}

168 Two papers (Srivastava \& Chaudhury, 2014; Bessell et al, 2012); were assessed using the Cochrane 169 risk of bias assessment tool which is suitable for assessing RCTs (Higgins \& Green, 2011). The Bessell

170 et al (2012) paper was assessed for risk of bias by two researchers independent of the paper's authors

171 (AM \& JG) as two of the authors were also the authors of this review.

172 Risk of Bias Assessment: Of the two papers, one (Bessell et al 2012) was found to be of low risk of bias

173 with regards to randomization sequence and allocation concealment (See table 1). Only one paper

174 was found to have low risk of bias for blinding of outcome assessor (Bessell et al 2012). All rates of

175 attrition were adequately documented in the papers. Srivastava and Chaudhury (2014) did not report

176 any attrition rates throughout the study period. All outcomes reported in the studies were reported in

177 the results.

178 Insert Table 1 here

179 RAMbo Assessment: Two papers (Jolly et al, 2010; Semple, Dunwoody, Kernohen \& McCaughan, 180 2009) were assessed using the RAMbo technique for observational studies (see Table 2). Jolly et al 181 (2010) did not report using a randomisation procedure, so was rated as unclear, whilst Semple et al, 1822009 did not use a randomisation technique so was rated at high risk of bias. Semple et al (2009) was 
183 rated at low risk of bias for attrition and measurement, whereas Jolly et al (2010) was rated as unclear

184 as multiple abstract publications of this study refer to different numbers of participants. The study

185 was also rated unclear for measurement as results for the anxiety outcome measure were not

186 reported.

187 Insert table 2 here

188 Effects of Interventions: Therapeutic approach

189 Cognitive-Behavioural Therapy: Jolly et al (2010) assessed the efficacy of an individual CBT program

190 for patients with lupus. The intervention focused on body image education, self-esteem, anxiety and

191 depression and also contained cosmetic training. The study employed 15 women with lupus (10

192 treatment and 5 controls) through a clinic in the United States. The mean ages of the participants in

193 the treatment and control groups were 43.6 years and 39.3 years respectively. Outcome measures

194 included Multi-Dimensional Body Relations Satisfaction - Appearance Scale (MBRSQ-AS), Situational

195 Inventory of Body Image Dysphoria (SIBID-SF), Body Image in Lupus Screen (BILS) and Anxiety and

196 Lupus PRO (Table 3).

197 Insert Table 3 here

198 The previous review by Bessell and Moss (2007) did not include meta-analyses. The authors of the

199 current review revisited the data from previous papers with a view to conducting meta-analyses on

200 any studies that consisted of randomised trials. Two of the original papers met this criterion

201 (Papadopoulos, Walker \& Anthis, 2004; Newell \& Clarke 2000). The Newell and Clarke (2000) paper

202 did not contain sufficient detail for meta-analysis. No other CBT studies consisted of randomised

203 trials, so it was not possible to conduct a meta-analysis on this intervention type. Overall the review

204 concluded there was very limited evidence for the efficacy of CBT for adults with visible differences. 
206 Combined CBT and SST: Bessell et al (2012) assessed the efficacy of two psychosocial interventions

207 against a no-treatment control. The first intervention consisted of a face-to-face CBT/SST

208 intervention, whilst the second was an online delivery of the same intervention model. The study

209 employed 83 individuals with varying visible differences recruited through charity organizations, the

210 Royal Free Hospital, London outpatient plastic surgery clinic and general advertising. Participants (34

211 male, 49 female) were over 18 years of age, with a mean age of 45 years (see Table 2 for study

212 information). Outcome measures used included the Hospital Anxiety and Depressions Scales (HADs),

213 the Derriford Appearance Scale-24 (DAS-24), and the Body Image Quality of life Inventory (BIQLI).

214 Semple et al (2009) assessed the efficacy of an individual CBT/SST program for patients with head and

215 neck cancer. The intervention focused on a series of specific areas including anxiety, depression,

216 fatigue, appearance and stress. The study employed 54 patients with head and neck cancer recruited

217 through the Regional head and Neck service in Northern Ireland. Participants (40 males, 14 females)

218 were 31 to $75+$ years of age. Outcome measures included the HADs, the Work and Social Adjustment

219 (WASA) scale and a health-related quality of life measure (University of Washington quality of life

220 scale version 4) which contained a measure of appearance-related distress.

221 The Semple et al (2009) paper did not contain sufficient detail to allow a meta-analysis to be

222 conducted. No other CBT studies consisted of randomised trials, so it was not possible to conduct a

223 meta-analysis on this intervention type. Overall the review found only very limited evidence for the

224 efficacy of a combined CBT and SST approach for adults with visible differences.

225 Person-centred: Srivastava and Chaudhury (2014) compared treatment as usual (one counselling

226 session; 83 participants) against a six session psychotherapeutic program (90 participants). 
227 Participants were aged $22-52$ years of age with a mean age of 30.05. All patients had experienced

228 amputation. Intervention consisted of six session based on reassurance, ventilation of emotions,

229 acceptance of self, therapeutic milieu md reintegration.

230 A study previously cited in the Bessell and Moss (2007) review also assessed the efficacy of a person-

231 centred approach (Papadopoulos et al, 2004). However this study did not contain enough information

232 to allow a meta-analysis to be conducted. Overall this review has found little evidence for the use of

233 the person-centred approach to therapy.

\section{Effects of Interventions: Method of Delivery}

235 Self-help: One of the included studies assessed the efficacy of self-help interventions. The Bessell et al

236 (2012) paper compared face-to-face delivery of a CBT intervention against an online delivery with

237 minimal facilitation from an assistant psychologist or counsellor.

238 Face-to-face individual: All four studies assessed the efficacy of individual CBT-based interventions.

239 The Bessell et al (2012) paper also assessed the efficacy of a face-to-face delivery of a CBT/SST

240 intervention administered by a trained counsellor or an assistant psychologist. The Semple et al

241 (2009) paper assessed a face-to-face CBT/SST intervention administered by a trained clinical nurse

242 specialist. Jolly et al (2010) assessed the efficacy of individual CBT-based support for women with

243 lupus. Srivastava and Chaudhury (2014) assessed the efficacy of individual psychotherapy delivered by

244 a psychiatric nurse for individuals with amputations.

246 Due to the differences in methodological design, it was difficult to draw any firm conclusions about

247 the optimal delivery of psychosocial interventions. Therefore, the review cannot recommend whether

248 any particular individuals should be responsible for delivering these psychosocial interventions. 


\section{Effects of Interventions: Timing of Intervention}

250 This review attempted to identify the optimal duration and intensity of intervention. The studies

251 included within this review varied in duration from two sessions (Semple et al, 2009), through to 10

252 sessions (Jolly et al, 2010). Full details of intervention duration can be found in Table 3 . The intensity

253 of the interventions consisted of weekly (Srivastava \& Chaudhury, 2014; Bessell et al, 2012) or

254 fortnightly sessions (Semple et al, 2009). Sessions were between one and two hours in length (see

255 Table 3 for full details of intensity).

256 Due to the differing intensity and duration across the studies, it is difficult to draw any firm

257 conclusions regarding the optimal length and intensity of therapy. However, most studies opted for

258 between $6-10$ sessions administered weekly for 1-1.5 hours. Therefore, it would seem reasonable to

259 conclude that this is the minimum intensity and duration required to lead to clinically significant

260 changes in appearance-related distress and anxiety. This also matches recommendations for the

261 minimum intensity of therapies in the general population (Roth \& Fonagy, 2005).

\section{Effects of Interventions: Participant Acceptability}

263 As well as assessing efficacy of interventions, it is important that trials of interventions also measure

264 patient acceptability. One paper reported on overall acceptability (Bessell et al, 2012; Newell \& Clark,

265 2000). The Bessell et al (2012) provided information about overall acceptability, as well as ratings of

266 usefulness and satisfaction for both the face-to-face and computer-based intervention. Users of the

267 face-to-face intervention gave it an average usefulness rating of 8.23 out 10 and a satisfaction rating

268 of 8 out 10 . The computer intervention was given ratings of 8.79 and 8.38 out of 10 respectively.

269 Overall acceptability for the face-to-face intervention was 51.89 out of 60 and 52.7 out 60 for the

270 computer intervention. The original Bessell and Moss (2007) review also included a study by Newell 
271 and Clarke (2000) which measured patient acceptability (not included in the previous review). Newell

272 and Clark (2000) paper found that $68.75 \%$ found the leaflet useful. Only $9.38 \%$ rated the booklet as

273 unhelpful. These papers suggest that the CBT or combine CBT and SST approach may be viewed as

274 acceptable by adults with visible differences.

275

276 Discussion

277 Main findings

278 The strength of the evidence to support the efficacy of the existing interventions from this narrative

279 synthesis is generally poor. The methodological quality of the included studies was limited and small

280 intervention effect sizes were observed. The studies looked at differing interventions making

281 judgments about consistency across studies difficult because each study used different intervention

282 settings, e.g. group, self-help or face-to-face and paradigms, e.g. CBT, SST or person-centred. There is

283 some very limited evidence to support the efficacy of a combined CBT and SST approach, but this is

284 far from conclusive as it is based on a combined sample size of 137 participants.

285 The length of intervention required was unclear with studies ranging from six to 10 sessions. No

286 firm conclusions can be made regarding the optimum therapy time required to reduce psychosocial

287 difficulties, or the most appropriate setting for these interventions. Neither can conclusions be drawn

288 about the level of therapist contact or expertise required to produce optimum results. Due to the

289 wide-ranging use of therapeutic paradigms of each intervention, it was not possible to draw any firm

290 conclusions regarding the acceptable content of psychosocial interventions for the visibly different

291 population, or the adequate implementation of these interventions. The participant populations were 
292 also varied in terms of conditions and symptom severity. Further studies need to be conducted to

293 establish which interventions are most effective for specific sub-populations.

295 Interpretation of findings in relation to previously published work

296 The findings of this review were no different to the conclusions of the original review (Bessell \&

297 Moss, 2007), which made recommendations for a greater number of future studies, including more

298 RCTs and experimental studies. Furthermore the need for greater methodological vigour was

299 highlighted with regards to ITT analyses, greater detail pertaining to attrition characteristics, rates

300 and causes, greater sample sizes, clearer inclusion and exclusion criteria, and studies that measure

301 interventions against control groups as standard. The review also emphasized the need for patient

302 acceptability ratings.

303 Eight years on from the publication of the original review and it would appear that little has

304 changed within this research field. The authors of this update decided to use a tighter inclusion

305 criteria than used previously to ensure only studies that measured body image or appearance-related

306 distress were included within the analysis. This limited the number of new studies to just four. This is

307 of fundamental importance. A recent review of self-help literature (Muftin \& Thompson, 2013)

308 included all interventions that focused on any condition that may be considered a visible difference.

309 These studies employed various outcome measures, but many did not include a direct measure of

310 appearance-related distress or body image. As this is the key variable in measuring level of distress in

311 this population (Clarke et al, 2008), it is important that future interventions measure this factor as

312 their primary outcome. 
313 Furthermore, of the four new studies included in this update, only one consisted of a RCT

314 reported in sufficient detail for low risk of bias and suitable for data pooling (Bessell et al, 2012). As

315 this study was conducted by the two of the authors of this review demonstrates how important this

316 timely update is for reminding future researchers of the importance of rigorous experimental design.

317 Current practice involves very limited testing of the efficacy of interventions, and this needs to be

318 addressed. In the modern health arena increasing focus is being placed on evidence-based practice

319 (Anderson, 2006). It is important that future research provides the necessary level of rigour in order

320 to establish such evidence-based practice to ensure clinicians can work with interventions known to

321 be effective for the target population.

322 Strengths and Limitations of this study

323 Credit must be given to the existing studies for trying to evaluate interventions for such a hard-to-

324 reach population. Designing interventions specifically for certain conditions classified as affecting

325 appearance can be very difficult due to the rarity of some conditions. Even when designing

326 interventions for a wide range of conditions, the population can still be difficult to reach leading to

327 low sample sizes and the population can vary widely, making generalizability a problem. Therefore

328 this review was based on small populations and meta-analysis was not possible due to differences in

329 study design. Future research needs to consider the use of multi-site studies in order to recruit larger

330 numbers of participants and thus increase the reliability of the findings of such evaluations.

332 Implications for policy and practice

333 It must be emphasized that despite the methodological problems associated with assessing these

334 interventions, the techniques themselves are still important. There is minimal service provision for 
335 individuals with visible differences (Bessell \& Moss, 2007) and yet there is a large population in need.

336 It is estimated that over one million in the United Kingdom alone have some form of visible

337 difference (Rumsey \& Harcourt, 2012), and of these approximately over one third experience

338 moderate to severe levels of distress (Rumsey et al, 2004). Although their efficacy still needs further

339 establishment, these interventions are necessary for increasing service provision for individuals with

340 visible differences. They are also needed to address the issue of an overall package of care for visibly

341 different clients from medical treatment right through to adjustment and psychosocial functioning.

342 For these reasons, further testing of these interventions is a fundamental step.

343 The current interventions have provided very limited support for the CBT and combined CBT and

344 SST models. These techniques offer individuals practical solutions to some of their social difficulties

345 without pathologising them. It is important that clinicians look to the small number of RCTs that have

346 been conducted in this area to guide their choice of therapeutic approach with clients with visible

347 differences. Currently these RCTs consist of ones that assess CBT (Newell \& Clarke 2000;

348 Papadopoulos et al, 2004) and those that assess a combined social skills and CBT approach (Bessell et

$349 \mathrm{al}, 2012)$. This is in keeping with the literature for body image issues in the general population, where

350 CBT-based approaches have been found to be most effective (Jarry \& Ip. 2005).

351 Furthermore, evidence from the acceptability measures used in some of the studies that involved

352 these approaches has suggested that individuals with visible differences do find these types of

353 interventions acceptable (Bessell et al, 2012; Newell \& Clarke, 2000). This is further supported by a

354 felt needs assessment recently conducted with potential service users within the field of visible

355 difference, which identified that most service users found the idea of CBT or SST to be acceptable and 356 positive (Bessell et al, 2010). This is an interesting point to note as it demonstrates that individuals 
357 with visible differences do not find the idea of interventions associated with their appearance

358 stigmatizing, as has often been a concern by experts in the past.

359

360 Conclusion

361 Overall this review concludes that to date there is very limited evidence to support the efficacy of CBT

362 or a combined CBT and SST approach for supporting adults with visible differences. However, there is

363 still insufficient information to draw firm conclusions and little to no information available regarding

364 the optimal setting for interventions of this nature, the optimal service provider, length of time or

365 intensity of intervention. These are all areas that future research needs to address. All these factors

366 must be addressed in order to demonstrate efficacy in the future. Furthermore, researchers and

367 clinicians alike must ensure key outcome measures are included when administering or testing

368 psychosocial interventions. In this instance some measure of body image or appearance-related

369 distress is important to ensure that interventions are specifically targeting appearance-related

370 factors. The authors conclude that little has changed in the research community since the publication

371 of the initial review. It is important that future research follows the recommendations made within

372 these reviews in order to provide strong evidence-based practice recommendations for clinicians in

373 the future.

374 
375

376

377

378

379

380

381

382

383

384

385

386

387

388

389

390

391

392

393

394

395

\section{References}

Altabe, M., \& Thompson, J.K. (1996). Body Image: A cognitive self-schema construct. Cognitive Therapy and Research, 20, 171-193.

Anderson, N.B. (2006). Evidence-based practice in psychology. American Psychologist, 61(4), 271-285.

Baumeister, R.F., \& Leary, M.R. (1995). The need to belong: Desire for interpersonal attachments as a fundamental human motivation. Psychological Bulletin, 117, 497-529.

Bessell, A., Brough, V., Clarke, A., Harcourt D., Moss T., \& Rumsey, N. (2012). Evaluation of the effectiveness of Face IT, a computer-based psychosocial intervention for disfigurement-related distress. Psychology, Health and Medicine, 5, 565-577.

Bessell, A., Clarke, A., Harcourt, D., Moss, T.P., \& Rumsey, N. (2010). Incorporating user perspectives in the design of an online intervention tool for people with visible differences: Face IT. Behavioural and Cognitive psychotherapy, 38, 577-596.

Bessell, A., \& Moss, T.P. (2007). Psychosocial interventions for visible differences: A systematic review. Body Image, 4, 227-238.

Bull, R., \& Rumsey, N. (1988). The social psychology of facial appearance. New York: Springer Verlag.

Cash, T.F. (2001). The body image workbook. Oakland CA: New Harbinger Publications.

Cash, T.F. (1996). The treatment of body image disturbances. In K. Thompson (Ed.), Body image, eating disorders and obesity. Washington, DC: American Psychological Association.

Chen, J.F., \& Wang, C.F. (2009). Introduction of critical appraisal of medical literature. Medical Dispute Mediation, 42, 19-24.

Clarke, A. (1999). Everybody's staring at me! London: Changing Faces. 
396 Clarke, A., Hansen, E., White, P., \& Butler, P.E.M. (2008). Psychological distress is high and impacts negatively on behaviour: Evidence from 1000 cosmetic referrals surgery in the NHS. Presentation at BAPRAS winter Scientific Meeting, Royal College of Surgeons, London, December.

Dixon-Woods, M., Agarwal, S., Jones, D., Young, B., \& Sutton, A. (2005). Synthesising qualitative and

Goffman, E. (1968). Stigma. London: Penguin books quantitative evidence: a review of possible methods. Journal of Health Services Research \& Policy, 10:45-53.

Higgins, J.P.T., \& Green, S. (2011). Cochrane handbook for systematic reviews of interventions. Oxford: The Cochrane Collaboration.

Jarry, J.L., \& Ip, K. (2005). The effectiveness of stand-alone cognitive-behavioural therapy for body image: A meta-analysis. Body image, 2, 317-331.

Jolly, M., Peters, K., Cash, T.F., Mikolaitis, R.A., Gao, W., \& Block, J.A. (2010). Body Image intervention improves health outcomes in systemic Lupus Erythematosus. Poster session presented the 416 Lupus Conference: 9th International Congress, Vancouver, CA.

Kent, G. (2000). Understanding experiences of people with disfigurement: An integration of four models of social and psychological functioning. Psychology, Health and Medicine, 5, 117-129.

Kleve, L., \& Robinson, E. (1999). A survey of psychological needs in adult burn-injured patients. Burns: Journal of the International Society for Burn Injuries, 25, 575-579.

Marcusson, A., Paulin, G., \& Ostrup, L. (2002). Facial appearance in adults who had cleft lip and palate treated in childhood. Scandinavian Journal of Plastic and Reconstructive Surgery and Hand Surgery, 36, 16-23. 
418 Muftin, Z., \& Thompson, A.R. (2013). A systematic review of self-help for disfigurement: Effectiveness, 419 usability and acceptability. Body Image, 10, 442-450.

420 Newell, R., \& Clarke, M. (2000). Evaluation of a self-help leaflet in treatment of social difficulties

421 following facial disfigurement. International Journal of Nursing Studies, 37, 381-388.

422 Newell, R., \& Marks, I. (2000). Phobic nature of social difficulty in facially disfigured people. British $423 \quad$ Journal of Psychiatry, 176, 177-181.

424 Papadopoulos, L., Walker, C., \& Anthis, L. (2004). Living with vitiligo: A controlled investigation into 425 the effects of group cognitive-behavioural and person-centred therapies. Dermatology and 426 Psychosomatics, 5, 172-177.

427 Roth, A., \& Fonagy, P. (2005). What works for whom? (2 ${ }^{\text {nd }}$ ed): A critical review of psychotherapy 428 research. New York: The Guilford Press.

429 Rumsey, N., Clarke, A., White, P., Wyn-Williams, M., \& Garlick, W. (2004). Altered body image: Auditing the appearance related concerns of people with visible disfigurement. Journal of Advanced Nursing, 48, 443-453.

432 Rumsey, N., \& Harcourt, D. (2012). The Oxford handbook of the psychology of appearance. Oxford: $433 \quad$ Oxford University Press.

434 Rumsey, N., Robinson, E., \& Partridge, J. (1993). An evaluation of the impact of social skills training for 435 facially disfigured people. Bristol: Changing Faces.

436 Semple, C., Dunwoody, L., Kernohan, W.G., McCaughan, E. (2009). Development and evaluation of a 437 problem-focused psychosocial intervention for patients with head and neck cancer. Support $438 \quad$ Care Cancer, 17(4), 379-388. 
439 Srivastava, K., \& Chaudhury, S. (2014). Rehabilitation after amputation: Psychotherapeutic 440 intervention module in Indian scenario. Scientific World Journal, doi: 10.1155/2014/469385.

441 
Figure 1 (on next page)

Flow diagram of search results 


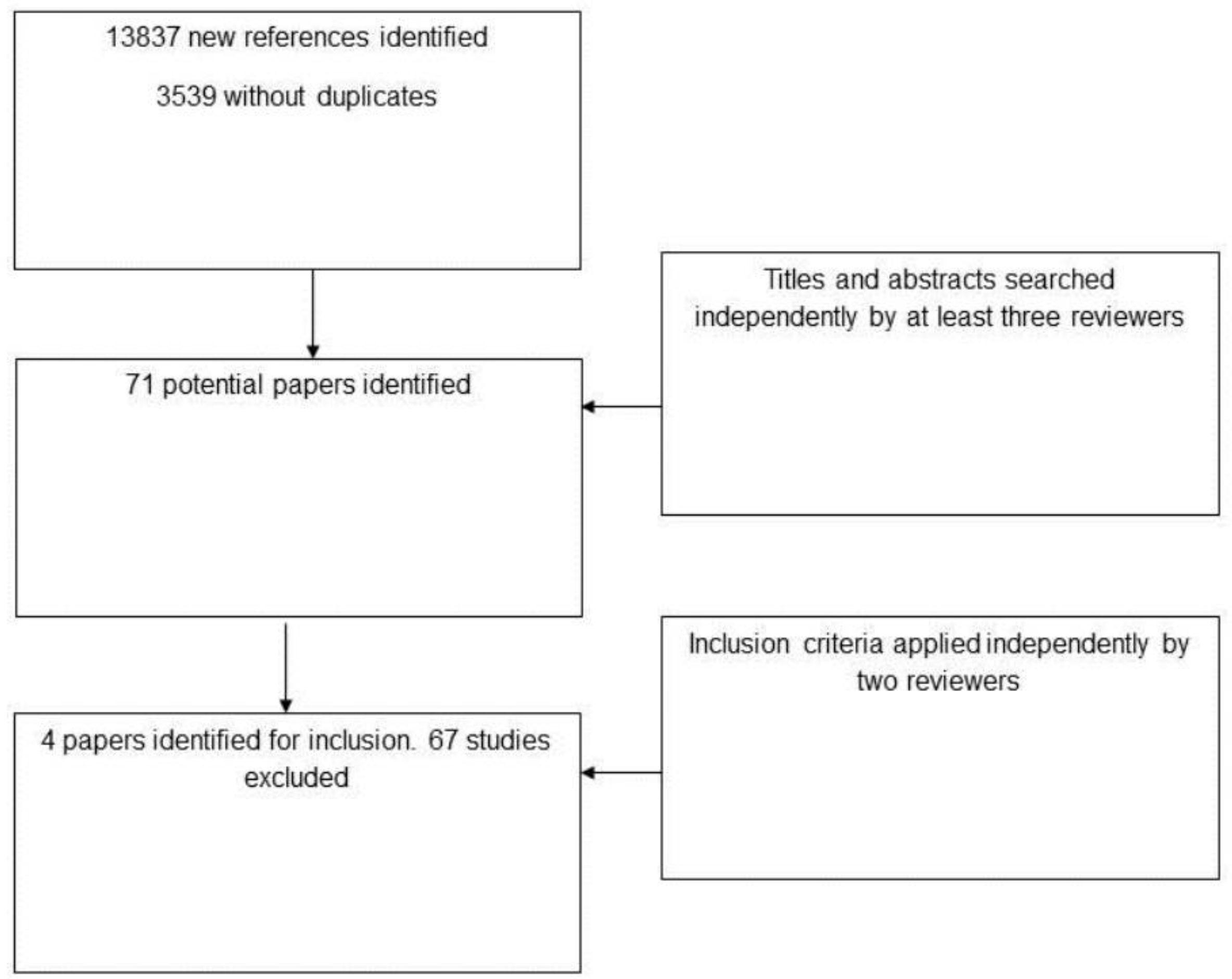




\section{Table 1 (on next page)}

Risk of bias in RCTs 


\section{Table 1: Risk of bias in RCTs}

\begin{tabular}{|c|c|c|c|c|c|c|}
\hline Study & $\begin{array}{l}\text { Study } \\
\text { Design }\end{array}$ & $\begin{array}{l}\text { Sequence } \\
\text { Generation }\end{array}$ & $\begin{array}{c}\text { Allocation } \\
\text { Concealment } \\
\end{array}$ & $\begin{array}{l}\text { Method of blinding of } \\
\text { outcome assessor }\end{array}$ & $\begin{array}{c}\text { Completeness of } \\
\text { outcome data } \\
\end{array}$ & $\begin{array}{l}\text { Reporting of } \\
\text { outcome data }\end{array}$ \\
\hline $\begin{array}{l}\text { Bessell et al (2012) } \\
\text { Srivastava \& }\end{array}$ & RCT & Low & Low & Low & Low & Low \\
\hline Chaudhury (2014) & RCT & Unclear & Unclear & Unclear & Low & Low \\
\hline
\end{tabular}

RCT = Randomised controlled trial, Low = low risk of bias, High = high risk of bias, unclear = information in the paper not sufficient to assess risk of bias 


\section{Table 2 (on next page)}

Risk of bias observational studies 
Table 2: Risk of bias observational studies

\begin{tabular}{lcccc}
\hline Study & Study Design & $\begin{array}{c}\text { Randomisation } \\
\text { Procedure }\end{array}$ & Attrition & Measurement \\
\hline Semple et al (2009) & Observational & High & Low & \\
Jolly et al (2010) & Observational & Unclear & Unclear & Unclear
\end{tabular}

Low $=$ low risk of bias, High $=$ high risk of bias, unclear $=$ information in the paper not sufficient to assess risk of bias 


\section{Table 3(on next page)}

Characteristics of included studies 
Table 3: Characteristics of included studies

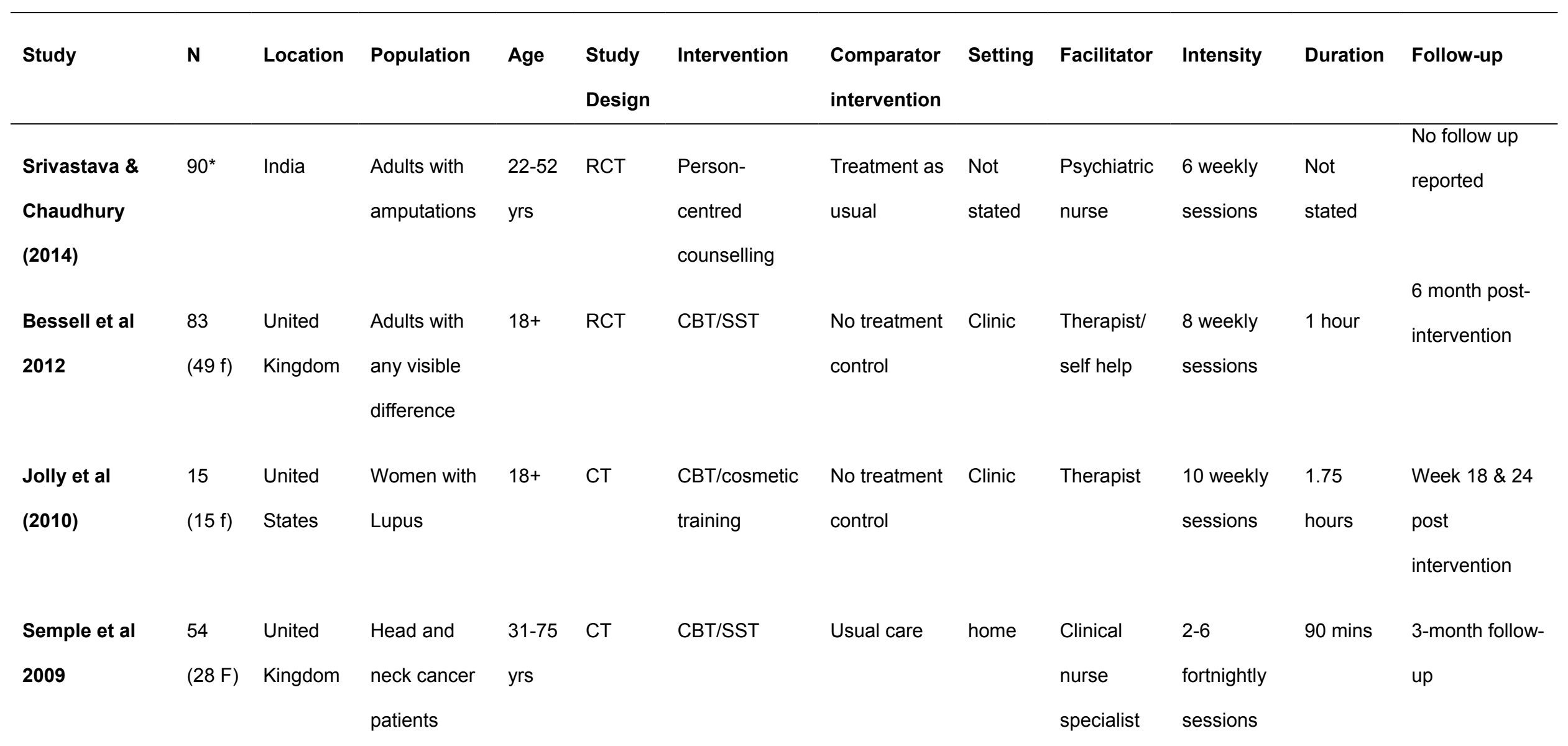

*Not all studies reported gender. Figures are provided where reported 